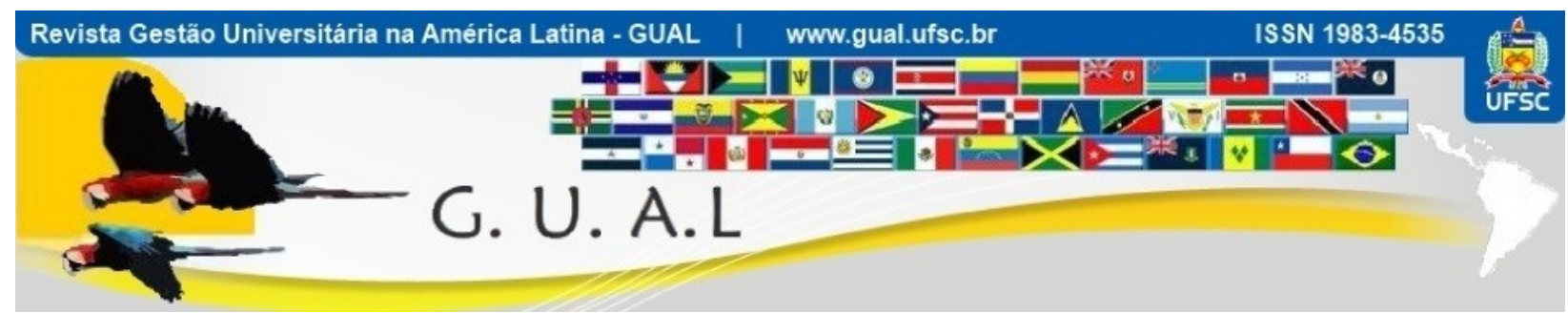

DOI: http://dx.doi.org/10.5007/1983-4535.2019v12n3p173

\title{
INOVAÇÃO ORGANIZACIONAL EM COMPRAS PÚBLICAS PELO SRP: ANÁLISE DA ACURÁCIA DO PLANEJAMENTO PARTICIPATIVO DO INSTITUTO FEDERAL DO TRIÂNGULO MINEIRO (IFTM)
}

\section{ORGANIZATIONAL INNOVATION IN PUBLIC PURCHASE BY SRP: ANALYSIS OF THE ACCURACY OF THE PARTICIPATIVE PLANNING OF THE FEDERAL INSTITUTE OF TRIÂNGULO MINEIRO (IFTM)}

Ricardo Oliveira Avigo, Mestre http://orcid.org/0000-0002-7721-5929 ricardoavigo@gmail.com Universidade Federal do Triângulo Mineiro | Mestrado Profissional em Administração Pública Uberaba | Minas Gerais | Brasil

Iraci de Souza João-Roland, Doutora https://orcid.org/0000-0003-3600-1091 iraci.joao@unifesp.br Universidade Federal de São Paulo | Instituto de Ciência e Tecnologia São José dos Campos | São Paulo | Brasil

Recebido em 11/setembro/2018

Aprovado em 08/abril/2019

Publicado em 02/setembro/2019

Sistema de Avaliação: Double Blind Review 


\title{
RESUMO
}

O Sistema de Registro de Preços (SRP) tem sido amplamente utilizado pelos órgãos públicos no planejamento de suas compras. Contudo, um dos grandes desafios em sua utilização é evitar a superestimação dos quantitativos registrados, isto é, adequá-los às reais demandas do órgão. No intuito de tornar as demandas do SRP mais próximas da realidade, o IFTM promoveu uma inovação em seu processo de planejamento de compras a partir de 2013, tornando-o participativo. Essa pesquisa teve por objetivo avaliar a inovação implementada por meio da análise dos resultados da efetivação das demandas dos registros de preços, a fim de verificar se estes refletiram a demanda real. Para isto, realizou-se um levantamento de todos os itens do planejamento participativo no período de 2013 a 2016, comparando os quantitativos registrados com os efetivamente adquiridos, por meio do indicador GARP grau de aproximação entre o real e o planejado. Os dados foram submetidos ao teste de duas amostras de Kolmogorov-Smirnov e à escala GARP. Constatou-se que o planejamento participativo não atingiu o objetivo de aproximar as demandas do SRP às reais necessidades do órgão. Como propostas de melhoria, sugeriu-se: adoção do índice do período anterior como subsídio para planejamento de quantitativos; conciliação dos valores licitados com o orçamento; capacitação dos servidores envolvidos; ações de monitoramento e controle e revisão da política de uso do SRP.

Palavras-chave: Compras Públicas. Planejamento. Previsão de Demanda. Sistema de Registro de Preços. Inovação Organizacional.

\begin{abstract}
The System of Price Record (SRP) has been widely used by the public organisations when planning their procurement. However, one of the greatest challenges in its usage is to avoid an overestimation of the quantity registered, that is, adequate them to the real demands of the public organisation. In order to make the SRP demands closer to reality, the IFTM promoted an innovation in its process of purchase planning in 2013, making it participative. This research aimed to assess the implemented innovation by analysing the outcomes of the implementation of the price register demands, in order to verify is these registers reflected the real demand. In order to do this, a survey of all the participatory planning items was carried out in the period from 2013 to 2016, comparing the recorded amounts with those actually acquired using the index GARP - approximation degree between the real and the planned. The data were submitted by two samples test of Kolmogorov-Smirnov and the GARP scale. It was verified that the participative planning did not reach the aim of bringing closer the SRP demands to the real necessities of the public organisation. As a proposal for improvement, it was suggested: adoption of an index from the previous period as a subsidey to the quantity planning; conciliation of the planned amounts with the budget; qualification of the involved professionals; monitoring and control of activities and finally, review of the of SRP usage policy.
\end{abstract}

Keywords: Public Purchase. Planning. Demand Forecast. System of Price Record. Organizational Innovation. 


\section{INTRODUÇÃO}

A gestão das compras públicas tem se tornado um dos temas mais relevantes da atualidade. Dados extraídos do Painel de Compras Governamentais apontam que entre janeiro e dezembro de 2017 foram realizados 98.779 processos de compras dentro do sistema, que é utilizado pelos órgãos federais, os quais movimentaram $\mathrm{R} \$ 46.938 .635 .818,22$ (quarenta e seis bilhões, novecentos e trinta e oito milhões, seiscentos e trinta e cinco mil, oitocentos e dezoito reais e vinte e dois centavos) demostrando a importância de se analisar o tema em profundidade.

O Sistema de Registro de Preços - SRP tem sido uma ferramenta cada vez mais utilizada no planejamento das aquisições públicas, pois permite que os órgãos concentrem suas aquisições em um menor número de processos licitatórios, para os quais não há a necessidade de aquisição imediata e nem do total licitado (RAPOSO et al., 2016; PINHEIRO; BOSELLI, 2016).

Contudo, a baixa acurácia da previsão de demanda no SRP, percebida quando os itens e quantitativos licitados não são efetivamente adquiridos pode causar prejuízos à Administração Pública e seus fornecedores, o que leva à perda gradual da credibilidade do órgão junto ao mercado fornecedor e ao descrédito do SRP (JACOBY FERNANDES, 2013; VEIGA, 2015; PEIXOTO, 2016; RAPOSO et al., 2016; PINHEIRO; BOSELLI, 2016). O Tribunal de Contas da União (TCU, 2014), em pesquisa realizada a respeito de governança nas contratações públicas, apontou que $85 \%$ das unidades auditadas tinham deficiências no planejamento das aquisições.

$\mathrm{Na}$ tentativa de aproximar os quantitativos licitados de sua real demanda o Instituto Federal de Educação, Ciência e Tecnologia do Triângulo Mineiro (IFTM) implementou em 2013 uma inovação organizacional, por meio da qual o planejamento de compras passou a ser participativo. Conforme informa a instituição em seu Plano de Desenvolvimento Institucional - PDI para o período de 2014 a 2018, o planejamento participativo foi implantando com o objetivo de "tornar a elaboração da proposta orçamentária e o planejamento de compras mais fidedigno e garantir maior economicidade nas contratações, padronização dos itens adquiridos e celeridade nas licitações (IFTM, 2013, p. 175)".

A forma adotada pela instituição pesquisada - IFTM - para planejar os itens e quantitativos a serem licitados pode ser considerada uma inovação organizacional 
(HALVORSEN et al., 2005; OCDE, 2005) por introduzir no âmbito da instituição uma nova forma de condução do processo de planejamento de compras.

Diante da importância de se realizar a avaliação e o monitoramento das inovações implementadas (FERRAREZI; AMORIM; TOMACHESKI, 2010; SANTOS, 2017), a presente pesquisa objetiva analisar os resultados do planejamento participativo no período de 2013 a 2016, por meio da comparação entre os quantitativos licitados pelo órgão em questão e os quantitativos efetivamente adquiridos ao longo da vigência das atas de registro de preço, a fim de verificar se a inovação implementada contribuiu para uma maior acurácia da previsão de demanda.

As principais implicações teóricas dessa pesquisa estão relacionadas à discussão de um tema ainda pouco explorado na literatura sobre compras públicas: a realização de demandas previstas por meio do SRP. Dessa forma, a presente pesquisa contribui para o aprofundamento de discussões a respeito do tema, o qual é de grande relevância para a Administração Pública em geral. A pesquisa realizada colabora também com a literatura a respeito de avaliação de inovações na Administração Pública, pois fornece uma avaliação detalhada a respeito dos resultados de uma inovação implementada em um órgão público por meio de dados objetivos e ainda aponta alternativas para o aprimoramento do processo.

Este artigo está estruturado em cinco partes. Inicialmente, na introdução é apresentado o tema de pesquisa e a instituição escolhida. Em seguida, no referencial teórico, são discutidos os temas licitação, com foco na modalidade pregão, planejamento das contratações públicas, Sistema de Registro de Preços e inovação organizacional no setor público. Na sequência, é apresentado o método de pesquisa e em seguida, os seus resultados com a respectiva discussão. Por fim, são apresentadas as considerações finais.

\section{REFERENCIAL TEÓRICO}

\subsection{SISTEMA DE REGISTRO DE PREÇOS}

A utilização do Sistema de Registro de Preços - SRP representou uma importante inovação em compras públicas, no sentido de que fomentou uma transformação acentuada nos procedimentos de compra e representa, atualmente, uma das grandes alternativas da operacionalização mais ágil dentro do processo de compras (PAIM TERRA, 2018).

O SRP é um "conjunto de procedimentos para registro formal de preços relativos à prestação de serviços e aquisição de bens, para contratações futuras” (BRASIL, 2013, p.2). 
Segundo Pereira Júnior e Dotti (2010), nas licitações pelo Sistema de Registro de Preços, a principal diferença reside no objetivo da licitação, pois nas licitações tradicionais o processo licitatório visa escolher um fornecedor e uma proposta para contratação imediata; já no SRP escolhe-se fornecedor e proposta para contratações não especificadas e seriadas, que poderão ocorrer quantas vezes forem necessárias, de modo que a proposta selecionada fica à disposição da Administração durante a vigência do registro de preços.

Jacoby Fernandes (2013) aponta que, no SRP, há uma reciprocidade e uma flexibilidade na relação e nas obrigações da Administração e do fornecedor. A administração não é obrigada a comprar o que foi licitado, porém caso queira comprar somente poderá fazê-lo com o signatário do registro de preços; o fornecedor, por usa vez, se compromete a garantir o preço pelo período estipulado, salvo se comprovar alterações significativas nos custos dos insumos, hipótese em que o preço poderá ser revisto. A validade do registro de preços na esfera federal pode ser de até um ano (BRASIL, 2013).

Raposo et al. (2016) relatam que o SRP é uma das alternativas mais úteis e interessantes de que dispõe a Administração Pública para a gestão de contratações, e destacam que seu uso é preferencial, conforme disposto no Art. 15 da Lei 8.666/93 (BRASIL, 1993):

Art. 15. As compras, sempre que possível, deverão:

(...)

II - ser processadas através de sistema de registro de preços;

A utilização do Sistema de Registro de Preços permite um melhor planejamento das contratações públicas, uma vez que uma única licitação pode ser utilizada por um período de até um ano, o que reduz o número de processos licitatórios e promove uma maior agilidade no momento do pedido de compra. A Administração pode acionar o fornecedor quantas vezes forem necessárias dentro da vigência do registro de preços, o que reduz a necessidade de se manter produtos em estoque, permite que o orçamento seja utilizado de forma parcelada, já que no SRP a disponibilidade orçamentária somente é exigida no momento da aquisição (PEREIRA JÚNIOR; DOTTI, 2010; VEIGA, 2015; PEIXOTO, 2016; RAPOSO et al., 2016; PEIXOTO, 2016; PINHEIRO; BOSELLI, 2016). O quadro 1 apresenta as principais vantagens da utilização do SRP encontradas na literatura pesquisada: 
Quadro 1 Vantagens da utilização do SRP

\begin{tabular}{|l|l|}
\hline \multicolumn{1}{|c|}{ Vantagem } & \multicolumn{1}{c|}{ Autor(es) } \\
\hline Melhor gestão de estoques. & $\begin{array}{l}\text { PEREIRA JÚNIOR; DOTTI, 2010; MOTTA, 2011; VEIGA, } \\
\text { 2015; RAPOSO et al., 2016; PEIXOTO, 2016. }\end{array}$ \\
\hline $\begin{array}{l}\text { Redução do número de } \\
\text { processos licitatórios. }\end{array}$ & $\begin{array}{l}\text { VEIGA, 2015; RAPOSO et al., 2016; PEIXOTO, 2016; } \\
\text { PINHEIRO; BOSELLI, 2016. }\end{array}$ \\
\hline Maior agilidade nas compras. & $\begin{array}{l}\text { VEIGA, 2015; PEIXOTO 2016; RAPOSO et al., 2016; } \\
\text { PINHEIRO; BOSELLI, 2016. }\end{array}$ \\
\hline $\begin{array}{l}\text { Não obrigatoriedade de dotação } \\
\text { orçamentária. }\end{array}$ & VEIGA, 2015; PEIXOTO, 2016; PINHEIRO; BOSELLI, 2016. \\
\hline $\begin{array}{l}\text { Utilização parcelada do } \\
\text { orçamento. }\end{array}$ & VEIGA, 2015; PEIXOTO, 2016; PINHEIRO; BOSELLI, 2016. \\
\hline
\end{tabular}

Fonte: elaborado pelos autores.

Como desvantagens do SRP, Nunes e Dantas (2012) ao analisarem licitações feitas pelo sistema tradicional e pelo Sistema de Registro de Preços no âmbito do Superior Tribunal de Justiça concluíram que, dada a incerteza da contratação para os fornecedores, os preços cotados pelas empresas tendem a ser maiores para fornecimento de materiais por meio do Sistema de Registro de Preços.

A impossibilidade de prever todos os itens a serem adquiridos é outra desvantagem do Sistema de Registro de Preços, o que pode gerar a superestimação dos itens e quantitativos no SRP (PINHEIRO; BOSELLI, 2016). Pinheiro e Boselli (2016), ao analisarem o Sistema de Registro de Preços sob o ponto de vista do fornecedor, apontam como desvantagem a incerteza da contratação e de seus quantitativos e a desnecessidade de indicação de dotação orçamentária o que, apesar de representar vantagem para os órgãos públicos, prejudica os fornecedores que se veem sem saber quanto, quando e se irão realmente vender ou prestar o serviço licitado.

Em relação à aquisição de quantitativos mínimos no SRP, destaca-se a particularidade introduzida no âmbito do Estado do Paraná por meio da Lei ${ }^{\circ} 17.081$, de 09 de fevereiro de 2012 (PARANÁ, 2012) que obriga a aquisição de um quantitativo mínimo nos processos de compras. Segundo a referida legislação, o administrador público que utilizar ao SRP deve, na vigência da ata de registro de preços, adquirir no mínimo 65\% (sessenta e cinco por cento) dos bens definidos e estimados no processo de compra, salvo por motivo devidamente justificado.

No âmbito federal, o Art. 16 do Decreto 7.892/2013 (BRASIL, 2013) trata a respeito da não obrigatoriedade de contratação pela Administração quando da utilização do Sistema de 
Registro de Preços. Contudo, mesmo com essa liberdade de não ser obrigado a formalizar as contratações, não deve o órgão público licitar sem o devido planejamento de quantidades, já que a exigência do inciso II do $\S 7^{0}$ do Art. 15 da Lei 8.666/93 é de que as quantidades licitadas devem ser planejadas em função da utilização e consumo prováveis (PINHEIRO; BOSELLI, 2016).

O quadro 2 apresenta uma compilação de problemas apontados na literatura ocorridos quando as quantidades previstas no SRP são superestimadas.

Quadro 2 Problemas decorrentes da superestimação de quantitativos no SRP

\begin{tabular}{|l|l|}
\hline \multicolumn{1}{|c|}{ Problema } & \multicolumn{1}{c|}{ Autor } \\
\hline $\begin{array}{l}\text { Perda gradual da credibilidade do Estado junto aos } \\
\text { fornecedores }\end{array}$ & $\begin{array}{l}\text { JACOBY FERNANDES, 2013; VEIGA, 2015; } \\
\text { RAPOSO et. al, 2016. }\end{array}$ \\
\hline $\begin{array}{l}\text { Geração de falsa expectativa de lucro nos } \\
\text { fornecedores }\end{array}$ & VEIGA, 2015; PEIXOTO, 2016. \\
\hline $\begin{array}{l}\text { Tendência ao não cumprimento dos compromissos } \\
\text { pelos fornecedores }\end{array}$ & VEIGA, 2015; PEIXOTO, 2016. \\
\hline $\begin{array}{l}\text { Inibição da participação de empresas menores } \\
\text { (prejuízo à competitividade do certame) }\end{array}$ & VEIGA, 2015; RAPOSO et. al, 2016. \\
\hline $\begin{array}{l}\text { Aumento dos preços dos itens diante da incerteza } \\
\text { da contratação }\end{array}$ & $\begin{array}{l}\text { NUNES; DANTAS, 2012; RAPOSO et al., } \\
\text { 2016; PINHEIRO; BOSELLI, 2016. }\end{array}$ \\
\hline
\end{tabular}

Fonte: elaborado pelos autores.

Fenili (2015) aponta que, na etapa de planejamento, o órgão público deve justificar a demanda prevista e a quantidade a ser adquirida, por meio de algum procedimento de cálculo. Dessa forma, é importante que os órgãos públicos utilizem técnicas de previsão de demanda que minimizem a subjetividade no processo. Jacoby Fernandes (2013) aponta que as quantidades previstas no SRP devem refletir efetivamente o que será adquirido e apresentar uma honesta e real estimativa do órgão, para que o próprio SRP não fique desacreditado. Para o autor, é certo que a Administração tem chances de encontrar propostas mais vantajosas à medida que exibir aos licitantes maior acurácia entre os quantitativos previstos e efetivamente demandados em suas licitações pelo SRP.

\subsection{INOVAÇÃO ORGANIZACIONAL NO SETOR PÚBLICO}

O tema inovação tem ganhado cada vez mais importância na literatura, devido ao aumento do número de publicações, que ocorreu principalmente no período de 2009 a 2014, no qual estão concentradas $60 \%$ do total das publicações sobre o assunto (VRIES; BEKKERS; TUMMERS, 2015). 
De acordo com o Manual de Oslo (OCDE, 2005) a inovação pode ser considerada como a implementação de um novo produto, bem ou serviço, significativamente melhorado, ou um novo processo, um novo método de marketing ou de outra área organizacional que tenha impactos nas práticas de negócios, na organização do local de trabalho ou nas relações externas da organização. Koch e Hauknes (2005) relatam que a inovação no setor público pode incluir a produção de coisas materiais, mas diferentemente do setor privado, envolve de forma mais frequente a aplicação de coisas já existentes ou a prestação de serviços, acompanhada por mudanças organizacionais relacionadas a alguma política pública.

Halvorsen et al. (2005) apontam que as inovações no setor público podem ser classificadas em: (i) inovação de serviço, que corresponde à criação de novos serviços ou melhorias em serviços já existentes; (ii), inovação de processo, isto é, mudanças na forma de prestação de um serviço ou de produção; (iii) inovação administrativa e organizacional, a qual se dá por meio da implementação de novas políticas ou práticas organizacionais ou de alterações nas práticas e políticas existentes; (iv) inovação do sistema, que corresponde ao estabelecimento de novas organizações ou novos padrões de cooperação entre organizações; (v) inovação de concepção, relacionada à nova missão, visão, objetivos e estratégias e (vi) mudança radical de racionalidade, relacionada a mudanças na forma de pensar e agir da organização e de seus colaboradores.

Mesmo com o crescimento da importância da inovação ao longo dos anos, existem muitas dificuldades para o desenvolvimento de inovações no âmbito da administração pública. Scherer (2014) aponta que a maioria das barreiras são de ordem legal, administrativa, cultural ou política. Oliveira (2014) cita que a rigidez estrutural no setor público é bem maior do que na esfera privada, de modo que a estrutura organizacional é menos propensa a modificações, o que dificulta o processo de inovação no setor público.

Santos (2017) chama atenção para o fato de que nem todas as inovações serão bem sucedidas após seu início e que a percepção sobre o sucesso pode variar entre os atores organizacionais envolvidos, de modo que o sucesso da inovação é dependente do fato de ela ser adequadamente gerida por estes atores. Portanto, a inovação envolve riscos, inclusive o risco de não corresponder às necessidades de maneira adequada.

Ferrarezi, Amorim e Tomacheski (2010) apontam que o sucesso de uma inovação está ligado à sua institucionalização, ou seja, se dá quando a inovação deixa de ter ligação com uma pessoa em particular e passa a ter caráter institucional. Os autores citam ainda a 
importância das atividades de monitoramento e controle da implementação das inovações, atividades que tem impacto direto no resultado da inovação, no sentido de permitir ajustes tempestivos que irão proporcionar um melhor desempenho e aumentar as chances de sucesso da inovação.

\section{METODOLOGIA}

Classifica-se este estudo como exploratório, pois, conforme Vergara (2016), estudos desse tipo são aqueles realizados em áreas nas quais há pouco conhecimento acumulado, tendo como objetivo o aprofundamento na realidade do fenômeno estudado. Quanto à abordagem, a pesquisa é quantitativa, uma vez que seu foco é a análise dos quantitativos licitados pela instituição pesquisada por meio do Sistema de Registro de Preços, comparandoos com os quantitativos efetivamente adquiridos. (VERGARA, 2016).

A unidade de observação foi o Instituto Federal de Educação, Ciência e Tecnologia do Triângulo Mineiro - IFTM, instituição escolhida por propor uma inovação organizacional no âmbito das compras públicas, denominada planejamento participativo de compras e também por critérios de acessibilidade dos pesquisadores. Trata-se, portanto, de uma amostra não probabilística selecionada por acessibilidade (VERGARA, 2007).

Para a coleta de dados, foi realizada pesquisa no sítio institucional do Instituto Federal do Triângulo Mineiro, a fim de obter documentos referentes ao planejamento participativo e seu desempenho. A pesquisa documental, na concepção de Godoy (1995, p. 21) é aquela na qual se examinam materiais de naturezas diversas, os quais ainda não possuem tratamento analítico ou nos quais se pode fazer um reexame, no sentido de serem obtidas novas interpretações.

Foi realizada uma consulta ao Portal de Compras Governamentais para o levantamento do número total de licitações realizadas de forma compartilhada pelo Sistema de Registro de Preços no âmbito da instituição pesquisada, período de 2013 a 2016. Foram coletados os seguintes dados: quantidade de licitações pelo SRP, vigência dos registros de preços e quantidade de itens por licitação. Após esse levantamento, foi realizado o levantamento do quantitativo dos itens registrados no SRP e quantitativos efetivamente comprados durante a vigência do registro de preços. Trata-se, portanto, de dados secundários, uma vez que os mesmos estão contidos em um banco de dados (GIL, 2002). A coleta dos dados foi realizada entre os meses de dezembro de 2017 e abril de 2018 e as informações obtidas organizadas em 
planilhas do programa Microsoft Excel, a fim de calcular o índice de realização de demandas de cada item e de cada licitação.

$\mathrm{O}$ instrumento utilizado para aferir a acurácia da previsão de demanda nos registros de preços foi o Grau de aproximação entre o real e o planejado - GARP, proposto por Veiga (2015). Segundo o autor, o indicador afere a proximidade entre os quantitativos registrados e os quantitativos efetivamente comprados, por meio da seguinte equação: $G A R P=\frac{Q T E C}{Q T R}$, onde: GARP = grau de aproximação entre o real e o planejado; QTEC = quantidade total efetivamente comprada; QTR = quantidade total registrada.

O GARP foi escolhido como indicador para essa pesquisa por se tratar de uma ferramenta simples, de fácil implementação e interpretação e que possui um grande potencial em termos de controle e acompanhamento da demanda por materiais no setor público, por meio da utilização de dados disponíveis a qualquer pessoa, por meio do Portal de Compras Governamentais e já ter sido utilizado com sucesso em pesquisas anteriores sobre o tema (VEIGA, 2015; PEIXOTO, 2016).

O Teste de duas amostras de Kolmogorov-Smirnov foi utilizado para comparar os resultados do índice GARP da presente pesquisa com valores predefinidos para o índice, a fim de observar se os resultados obtidos com a inovação denominada planejamento participativo apresentam melhor desempenho quando comparados com os valores de referência, razão pela qual o teste foi adotado em sua forma unilateral (SIEGEL; CASTELLAN JR., 2006).

Para fins de realização do teste foram consideradas as seguintes hipóteses:

a) Hipótese nula $\left(\mathrm{H}_{0}\right)$ : a previsão de demanda do planejamento participativo reflete a real necessidade de consumo do órgão; e.

b) Hipótese alternativa $\left(\mathrm{H}_{1}\right)$ : a previsão de demanda do planejamento participativo não reflete a real necessidade de consumo do órgão.

Dessa forma, como a hipótese nula $\left(\mathrm{H}_{0}\right)$ é de que a previsão de demanda dos itens reflete a real necessidade do órgão, foram estabelecidos, para fins de comparação com os dados encontrados, dois cenários de valores de referência para o indicador GARP:

a) 0,65 , com base no valor mínimo de $65 \%$ de aquisição exigido na Lei $\mathrm{n}^{\circ}$ 17.081/2012 do Estado do Paraná (PARANÁ, 2012) que, embora não se aplique aos órgãos federais, representa um parâmetro de desempenho para a previsão de demanda no SRP; e

b) 0,60 , com base na escala do índice GARP proposta por Veiga (2015), para a qual os itens com GARP acima de 0,60 são considerados como de previsão de demanda "satisfatória"; 
O nível de significância considerado para o teste foi de 5\%.

O valor de $X^{2}$ foi determinado por meio da fórmula $X^{2}=4 D_{m, n}^{2} \frac{m n}{m+n}$, onde: $D_{m, n}^{2}=$ maior diferença positiva entre a frequência relativa encontrada e a frequência relativa predita; $m=$ número de respostas da amostra com os valores preestabelecidos; e $n=$ número de respostas da amostra com os valores observados. O valor de $X^{2}$ foi aproximado por uma distribuição qui-quadrado com dois graus de liberdade $(g l=2)$, a fim de se obter a significância do valor de $D_{m, n}$, por meio da tabela de distribuição do qui-quadrado (SIEGEL; CASTELLAN JR., 2006). A hipótese nula $\mathrm{H}_{0}$ foi rejeitada para os casos em que o valor de significância de $D_{m, n}$ foi menor ou igual $\alpha$.

Além do Teste de duas amostras de Kolmogorov-Smirnov, foi feita a análise dos resultados encontrados com base na escala GARP, criada por Veiga (2015) como forma de padronizar a análise do índice GARP, conforme figura 1:

Figura 1 Escala de análise do índice GARP

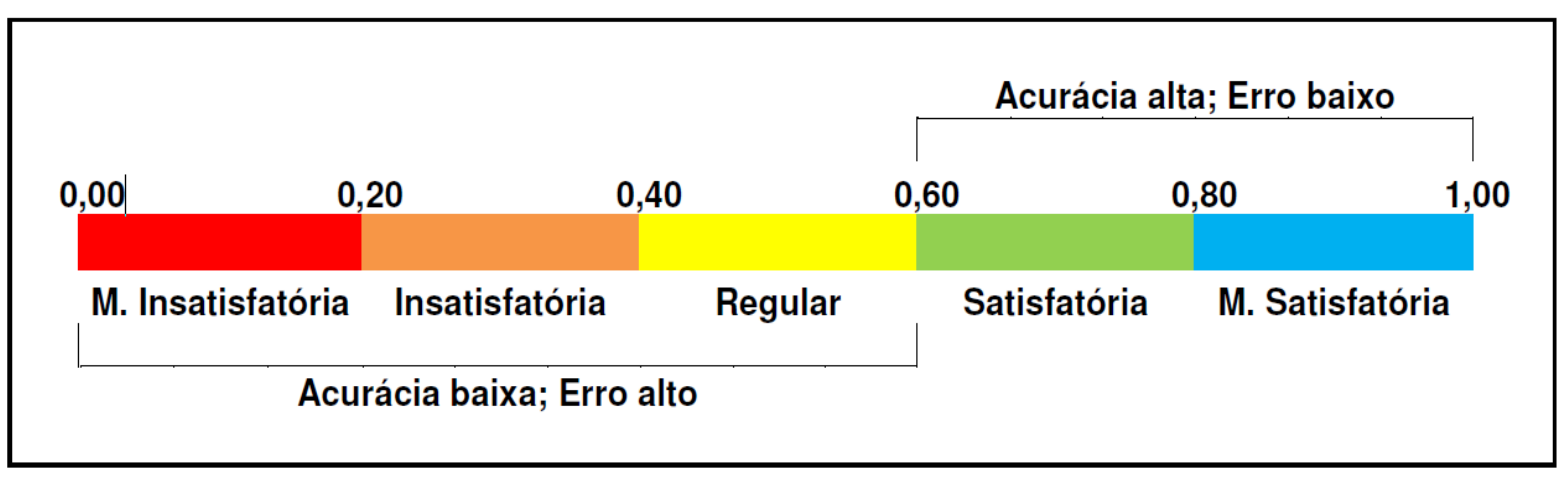

Fonte: Veiga (2015, p. 61).

Para fins de amostragem, a presente pesquisa considerou como objeto as licitações que atenderam a todos os requisitos a seguir: (i) realizadas entre 2013 e 2016; (ii) cuja vigência do Registro de Preços tenha expirado até 31/12/2017; (iii) realizadas pelo Sistema de Registro de Preços; e (iv) que tiveram seus quantitativos definidos pelo Planejamento Participativo. Vale ressaltar que a inovação organizacional analisada foi implementada a partir de 2013.

\section{RESULTADOS}

\subsection{O PLANEJAMENTO PARTICIPATIVO}

O processo de planejamento participativo no IFTM se dá, conforme descrito em seu PDI 2014-2018 (IFTM, 2013), da seguinte forma: a instituição foi dividida em áreas 
denominadas centros de custos, por meio das quais os servidores podem inserir em um sistema específico os itens que desejam que sejam adquiridos pelo órgão em suas licitações, conforme a necessidade de seus setores. Essas demandas são analisadas pelos Diretores Gerais dos campi e pelos Pró-Reitores (estes últimos apenas em relação aos centros de custos da Reitoria), os quais são responsáveis por promover os devidos ajustes em consonância com o orçamento e com as demais despesas do órgão. As demandas aprovadas são consolidadas e separadas em listas, de acordo com as naturezas das despesas, as quais são encaminhadas para licitação, com o objetivo de registrar preço para as aquisições (figura 2).

Figura 2 Fluxo do planejamento participativo

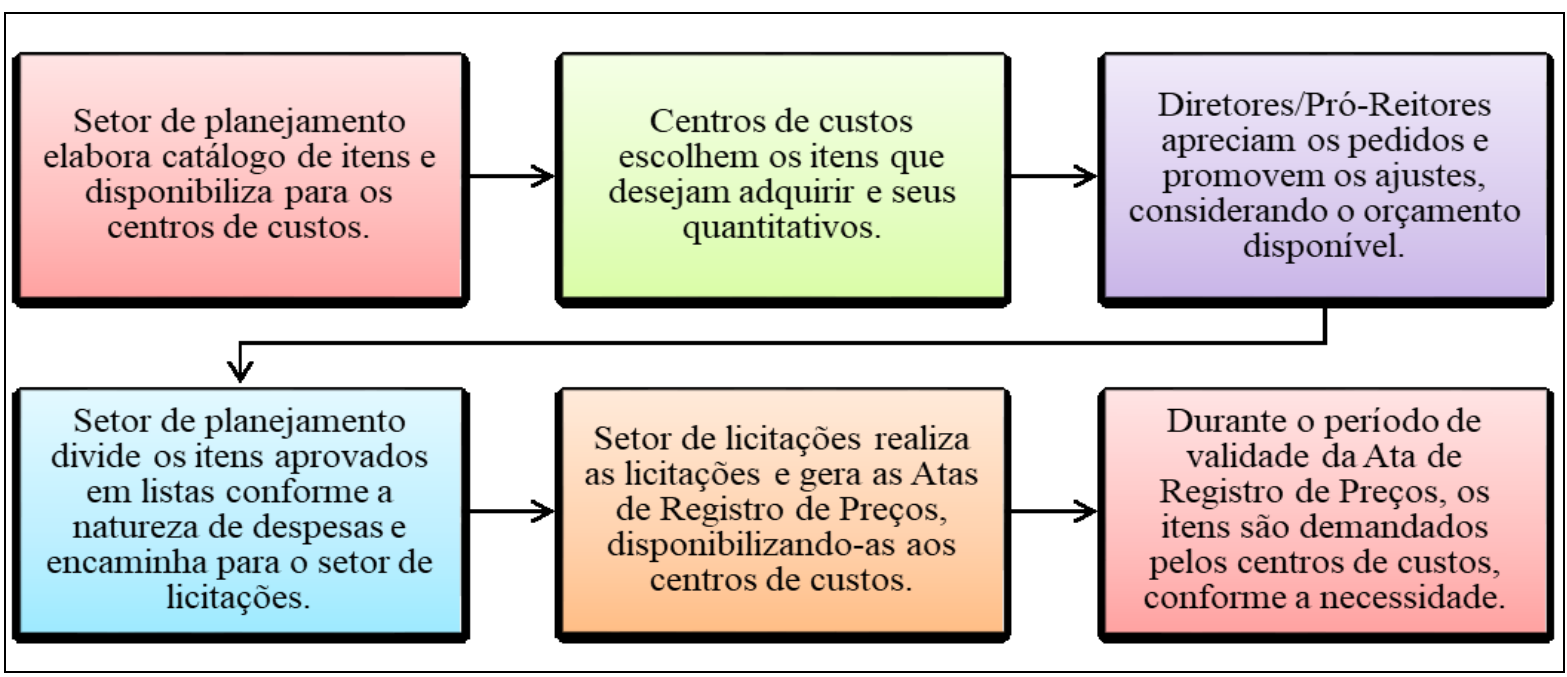

Fonte: elaborado pelos autores, a partir de IFTM (2013).

O fluxo do planejamento participativo é contínuo. A etapa de elaboração do catálogo de itens fica disponível durante todo o ano. Porém, as etapas de planejamento dos quantitativos pelos centros de custos e de apreciação dos pedidos pelos Diretores/Pró-Reitores têm prazos definidos para ocorrer, quarenta e cinco dias e duas a três semanas respectivamente.

Com a implementação do planejamento participativo houve uma mudança nas práticas de planejamento anteriores, no sentido de que se promoveu a descentralização do processo, à medida que mais servidores foram envolvidos no planejamento das compras e passaram a ter a responsabilidade de definir os itens e quantitativos a serem licitados. A mudança nas práticas anteriores, por meio da incorporação de novos elementos é uma das principais caraterísticas das inovações (OCDE, 2005).

Dentre as classificações propostas por Halvorsen et al. (2005) o planejamento 
participativo enquadra-se como uma inovação organizacional, pois teve como objetivo alterar, de forma substancial, o processo de planejamento de compras da instituição, por meio do envolvimento de um maior número de servidores, na tentativa de assim tornar a previsão da demanda mais próxima da real necessidade de consumo do órgão, conforme explicitado em seu PDI 2014-2018 (IFTM, 2013).

\subsection{LEVANTAMENTO DAS LICITAÇÕES}

Durante o período de análise, foram identificados 123 pregões realizados pelo Sistema de Registro de Preços oriundos do Planejamento Participativo de Compras, sendo 87 pregões $(70,7 \%)$ destinados à aquisição de materiais de consumo e $36(29,3 \%)$ destinados à aquisição de materiais permanentes.

Os valores dos registros de preços firmados pelo IFTM no período pesquisado somam R\$ 39.254.284,07 (trinta e nove milhões, duzentos e cinquenta e quatro mil, duzentos e oitenta e quatro reais e sete centavos). Do total de itens licitados no período, $74,74 \%$ tiveram seus preços registrados, e os demais foram cancelados durante a licitação. A tabela 1 apresenta a quantidade de itens com registro de preços concluído no período pesquisado, separando-os em material de consumo e material permanente.

Tabela 1 Itens com registro de preço concluído, por categoria

\begin{tabular}{lcccc}
\hline \multirow{2}{*}{ Categoria } & \multicolumn{3}{c}{ Quantidade de itens } & Percentual de \\
\cline { 2 - 4 } & Licitados & Cancelados & Registrados & registro \\
\hline Consumo & 10.777 & 2.562 & 8.215 & $76,28 \%$ \\
Permanente & 981 & 408 & 573 & $58,41 \%$ \\
\hline Total & 11.758 & 2.970 & 8.788 & $74,74 \%$ \\
\hline
\end{tabular}

Fonte: elaborado pelos autores, a partir de dados do Portal de Compras Governamentais.

Chama atenção o fato de que os materiais permanentes, apesar de menor participação, possuem um índice de cancelamento de itens consideravelmente superior ao dos materiais de consumo. Quanto aos motivos para a o cancelamento dos itens, Luchi e Carneiro (2006) apontam que os itens podem ser: desertos, quando não há propostas válidas; fracassados, quando as propostas válidas não foram classificadas; revogados, quando os itens são cancelados por razões de interesse público e anulados, quando os itens são cancelados por estarem com algum aspecto de ilegalidade.

Como os itens de materiais permanentes destinam-se normalmente a compra de equipamentos, os mesmos possuem características mais restritas e exigências maiores de 
garantia e assistência técnica, o que conduz, caso tais exigências não sejam atendidas pelos licitantes, ao cancelamento dos itens, o que explica o percentual maior de cancelamento nos itens de material permanente.

Considerando que a quantidade de itens cancelados foi de 2.970, o índice GARP e o teste de duas amostras de Kolmogorov-Smirnov foram aplicados a 8.788 itens, uma vez que os itens cancelados não possuem quantitativos registrados.

\subsection{ANÁLISE DA ACURÁCIA DO PLANEJAMENTO PARTICIPATIVO}

Os resultados do teste unilateral de duas amostras de Kolmogorov-Smirnov (tabela 2) demonstram que quando considerado o índice GARP predito de 0,65 , o valor de $D_{m, n}$ é de 0,000006, ou seja, se dá no sentido negativo, de modo que não foi possível prosseguir com o cálculo, uma vez que a maior diferença encontrada não se deu no sentido predito (que é o de aumento). Por essa razão, não aceitou-se $\mathrm{H}_{0}$ para o GARP mínimo predito de 0,65.

Tabela 2 Valor de $D_{m, n}$ para GARP predito de 0,65 e 0,60

\begin{tabular}{cc}
\hline Índice GARP predito & Valor de $\mathrm{D}_{\mathrm{m}, \mathrm{n}}$ \\
\hline 0,65 & $-0,000006$ \\
0,60 & 0,000091 \\
\hline
\end{tabular}

Fonte: elaborado pelos autores, a partir de dados da pesquisa.

Como para o GARP predito de 0,60 o valor de $D_{m, n}$ foi positivo $(0,000091)$ e o valor de $m$ e $n$ foi 8.788 (quantidade de itens pesquisados), foi calculado o valor de $\mathrm{X}^{2}$ :

$$
X^{2}=4 D_{m, n}^{2} \frac{m n}{m+n}=4 *(0,000091)^{2} * \frac{(8.788 * 8.788)}{(8.788+8.788)}=0,000145653
$$

A consulta à tabela de distribuição do qui-quadrado revela que a probabilidade associada com $\mathrm{X}^{2}=0,000145653$ para $g l=2$ é $p>0,99$ (teste unilateral). Como o valor de $p$ é maior que $\alpha=0,05$, rejeitou-se novamente $\mathrm{H}_{0}$ em favor de $\mathrm{H}_{1}$.

Dessa forma, a hipótese nula $\left(\mathrm{H}_{0}\right)$ foi rejeitada para as duas predições do índice GARP, 0,65 e 0,60, ao nível de significância $\alpha=0,05$.

A tabela 3 apresenta as possíveis decisões a respeito da hipótese nula $\left(\mathrm{H}_{0}\right)$, tendo como base valores estimados para o GARP predito, no intuito de demonstrar a partir de que predição do GARP $\mathrm{H}_{0}$ poderia ser aceita: 
Tabela 3 Simulações de decisão sobre $\mathrm{H}_{0}$ conforme GARP predito.

\begin{tabular}{cccccc}
\hline Índice GARP predito & Valor de $\boldsymbol{D}_{\boldsymbol{m}, \boldsymbol{n}}$ & Valor de $\mathrm{X}^{2}$ & $\boldsymbol{p}$-valor & $\boldsymbol{\alpha}$ & Decisão sobre $\mathbf{H}_{\mathbf{0}}$ \\
\hline 0,90 & $-0,000102$ & $\mathrm{NA}^{\mathrm{a}}$ & $\mathrm{NA}^{\mathrm{a}}$ & 0,05 & Rejeitada \\
0,80 & $-0,000091$ & $\mathrm{NA}^{\mathrm{a}}$ & $\mathrm{NA}^{\mathrm{a}}$ & 0,05 & Rejeitada \\
0,70 & $-0,000080$ & $\mathrm{NA}^{\mathrm{a}}$ & $\mathrm{NA}^{\mathrm{a}}$ & 0,05 & Rejeitada \\
0,60 & 0,000091 & 0,000145653 & $>0,99$ & 0,05 & Rejeitada \\
0,50 & 0,001061 & 0,019797411 & $>0,99$ & 0,05 & Rejeitada \\
0,40 & 0,009873 & 1,71317631 & $0,5>p>0,3$ & 0,05 & Rejeitada \\
0,30 & 0,036752 & 23,74067066 & $<0,001$ & 0,05 & Aceita \\
0,20 & 0,115538 & 234,6245205 & $<0,001$ & 0,05 & Aceita \\
0,10 & 0,213780 & 803,2527988 & $<0,001$ & 0,05 & Aceita \\
\hline
\end{tabular}

Fonte: elaborado pelos autores, a partir de dados da pesquisa.

a) NA (Não apurado): Como o valor de $D_{m, n}$ foi negativo para o GARP predito, não foi possível o cálculo do valor de $\mathrm{X}^{2}$ e os demais cálculos seguintes.

Conforme dados apresentados na tabela 3, a hipótese de que o planejamento participativo reflete adequadamente a demanda da instituição somente poderia ser aceita caso o índice GARP adotado fosse próximo de 0,30, o que indica que o índice de efetivação das demandas do planejamento participativo está próximo desse valor.

\subsection{RESULTADOS DA APLICAÇÃO DO ÍNDICE GARP}

A tabela 4 apresenta os resultados do índice GARP por ano, bem como a distribuição dos itens registrados em cada uma das categorias da escala proposta por Veiga (2015):

Tabela 4 Acurácia dos itens do período de 2013 a 2016.

\begin{tabular}{|c|c|c|c|c|c|c|c|c|}
\hline \multirow[b]{2}{*}{ Ano } & \multirow[b]{2}{*}{ GARP } & \multirow[b]{2}{*}{ Acurácia } & \multirow[b]{2}{*}{ Registros } & \multicolumn{5}{|c|}{ Classificação } \\
\hline & & & & $\begin{array}{l}\text { Muito } \\
\text { Insat. }\end{array}$ & Insat. & Regular & Satisf. & $\begin{array}{l}\text { Muito } \\
\text { Satisf. }\end{array}$ \\
\hline \multirow{2}{*}{2013} & \multirow{2}{*}{0,4095} & \multirow{2}{*}{ Regular } & \multirow{2}{*}{3.355} & 1.535 & 443 & 340 & 254 & 783 \\
\hline & & & & $45,75 \%$ & $13,20 \%$ & $10,13 \%$ & $7,57 \%$ & $23,34 \%$ \\
\hline \multirow{2}{*}{2014} & \multirow{2}{*}{0,2381} & \multirow{2}{*}{ Insatisfatória } & \multirow{2}{*}{1.614} & 1.102 & 128 & 96 & 55 & 233 \\
\hline & & & & $68,28 \%$ & $7,93 \%$ & $5,95 \%$ & $3,41 \%$ & $14,44 \%$ \\
\hline \multirow{2}{*}{2015} & \multirow{2}{*}{0,2418} & \multirow{2}{*}{ Insatisfatória } & \multirow{2}{*}{2.791} & 1.623 & 300 & 293 & 186 & 389 \\
\hline & & & & $58,15 \%$ & $10,75 \%$ & $10,50 \%$ & $6,66 \%$ & $13,94 \%$ \\
\hline \multirow{2}{*}{2016} & \multirow{2}{*}{0,2564} & \multirow{2}{*}{ Insatisfatória } & \multirow{2}{*}{1.028} & 571 & 126 & 123 & 64 & 144 \\
\hline & & & & $55,54 \%$ & $12,26 \%$ & $11,96 \%$ & $6,23 \%$ & $14,01 \%$ \\
\hline \multirow{2}{*}{ Total } & \multirow{2}{*}{0,2779} & \multirow{2}{*}{ Insatisfatória } & \multirow{2}{*}{8.788} & 4.831 & 997 & 852 & 559 & 1.549 \\
\hline & & & & $54,97 \%$ & $11,35 \%$ & $9,70 \%$ & $6,36 \%$ & $17,63 \%$ \\
\hline
\end{tabular}

Fonte: elaborado pelos autores, a partir de dados do Portal de Compras Governamentais. 
Considerando o GARP médio de 0,2779 encontrado, constata-se que o planejamento participativo não atingiu o objetivo de tornar as demandas mais fidedignas e próximas da real necessidade da instituição, uma vez que há uma grande disparidade entre os quantitativos registrados e os quantitativos efetivamente demandados durante a vigência das atas de registro de preço. Como o ano de 2013 foi o primeiro ano do planejamento participativo, o seu resultado melhor em relação aos demais anos analisados pode ser explicado pelo fato de ter havido treinamento inicial com os envolvidos, o que contribuiu para que a previsão da demanda tivesse melhor qualidade em relação aos anos seguintes.

Cumpre ressaltar ainda que mudanças na estrutura organizacional da instituição ocorridas entre 2013 e 2016 impactaram também em mudança nos responsáveis por centros de custos, ou seja, os servidores envolvidos na previsão de demanda nem sempre são os mesmos ao longo do período pesquisado. Como não houve replicação do treinamento ao longo dos anos, os servidores novos nos centros de custo podem estar com dificuldades em determinar quantitativos e/ou operar o sistema.

Outra razão para o baixo índice de realização das demandas pode estar associada à disparidade existente entre os valores licitados e os valores previstos no orçamento do órgão, isto é, se o órgão licita mais do que sua proposta orçamentária, certamente não terá condições de adquirir os quantitativos registrados, o que leva a um menor índice de realização das demandas.

\subsection{RELAÇÃO ENTRE OS VALORES LICITADOS E O ORÇAMENTO}

A tabela 5 apresenta a comparação entre os valores consignados por ano nas propostas orçamentárias para aquisição de materiais e os valores licitados para tais aquisições. Teixeira (2017) em trabalho que analisou a construção dos processos de controle orçamentário do IFTM constatou que, devido ao insucesso nas licitações de alguns itens, bem como a redução de preços após a disputa nas licitações, os servidores ocupantes de cargos de direção na instituição costumam permitir que os centros de custos planejem com uma margem de $20 \%$ a $30 \%$ acima do orçamento, para garantir a execução adequada.

Os resultados da presente pesquisa identificaram um percentual de itens cancelados de $25,26 \%$, condizente com os dados citados por Teixeira (2017). Contudo, conforme os valores levantados, verifica-se que, no período de 2013 a 2016 o órgão licitou 475,21\% de seu 
orçamento, o que pode ser usado para explicar a baixa acurácia nas previsões de demanda, conforme demonstrou este trabalho.

Tabela 5 Comparação entre o orçamento e os valores licitados

\begin{tabular}{|c|c|c|c|c|c|}
\hline Ano & $\begin{array}{c}\text { Proposta } \\
\text { Orçamentária (R\$) }\end{array}$ & $\operatorname{Licitado}_{(\mathbf{R} \$)^{1}}$ & $\begin{array}{l}\text { Percentual } \\
\text { licitado }\end{array}$ & $\begin{array}{l}\text { Resultado das } \\
\text { licitações }^{2} \text { (R\$) }\end{array}$ & $\begin{array}{c}\text { Percentual } \\
\text { final }\end{array}$ \\
\hline 2013 & $8.505 .114,00$ & $24.736 .108,15$ & $290,84 \%$ & $15.510 .407,98$ & $182,37 \%$ \\
\hline 2014 & $8.322 .999,00$ & $90.244 .042,46$ & $1.084,27 \%$ & $13.804 .030,93$ & $165,85 \%$ \\
\hline 2015 & $10.592 .054,20$ & $19.935 .749,79$ & $188,21 \%$ & $9.539 .777,19$ & $90,07 \%$ \\
\hline 2016 & $4.738 .638,00$ & $17.904 .881,71$ & $377,85 \%$ & $11.092 .187,04$ & $234,08 \%$ \\
\hline Total & 32.158.805,20 & 152.820.782,11 & $475,21 \%$ & 49.946.403,14 & $155,31 \%$ \\
\hline
\end{tabular}

Fonte: elaborado pelos autores, a partir de dados do Painel do Orçamento Federal e do sítio eletrônico do IFTM.

\subsection{DISCUSSÃO DOS RESULTADOS}

Diante dos resultados encontrados, constata-se que o planejamento participativo não tem funcionado adequadamente como uma ferramenta de previsão de demanda, uma vez que, conforme os resultados da pesquisa, no período pesquisado não houve melhoria do índice de realização de demanda em relação aos valores estabelecidos, ou seja, não se pode considerar que o planejamento participativo atingiu o objetivo de tornar as demandas de compras mais fidedignas, objetivo este previsto no PDI 2014-2018 do IFTM (IFTM, 2013).

No intuito de propor melhorias, sugere-se no presente trabalho algumas iniciativas que podem ser adotadas pela instituição pesquisada, no intuito de aperfeiçoar o planejamento de compras. A primeira delas é a utilização do índice de realização de demandas dos anos anteriores como base para o planejamento dos anos seguintes. Assim, será possível identificar os itens com baixa realização de demandas e ajustar o quantitativo destes para as próximas licitações, a fim de evitar que a superestimação dos quantitativos se repita de um ano para outro.

Como os valores licitados e registrados encontrados foram bastante superiores ao montante da proposta orçamentária, constatou-se haver deficiência na etapa de ajustes das demandas com o orçamento, que tem permitido o registro de quantitativos acima dos valores previstos no orçamento, os quais não são adquiridos.

\footnotetext{
${ }^{1}$ Considerou-se como valor licitado o valor das licitações divulgadas pelo órgão, antes da fase de disputa por preços.

${ }^{2}$ Considerou-se como valor dos resultados o valor apurado após o final das licitações, ou seja, após a fase de disputa por preços.
} 
Neste sentido, a fim de promover uma maior aproximação entre o orçamento disponível e os valores licitados, recomendou-se a adoção de um mecanismo de bloqueio de demandas que ultrapassem $130 \%$ do valor da proposta orçamentária, o que tornaria o ajuste por parte dos Diretores Gerais/Pró-Reitores obrigatório dentro deste limite. Dessa forma, o valor licitado ficará mais próximo da proposta orçamentária (considerando o percentual de insucesso de 24,26\% dos itens licitados), o que proporcionará um aumento do índice de realização das demandas.

Além disso, sugere-se a capacitação dos servidores envolvidos na realização de demandas no SRP, com foco em técnicas quantitativas de estimação, uma vez que estas devem ser usadas de forma preferencial, conforme previsto na Lei $\mathrm{n}^{\circ}$ 8.666/93 (BRASIL, 1993). Recomenda-se que as ações de capacitação sejam realizadas de forma periódica, de modo a promover uma maior conscientização dos servidores envolvidos na formulação de demandas a respeito de compras públicas e da importância de se realizar um planejamento adequado.

Diante da importância da avaliação e do monitoramento constante das inovações (FERRAREZI; AMORIM; TOMACHESKI, 2010) recomenda-se que a instituição execute de forma contínua ações de monitoramento e controle referentes às demandas originadas do planejamento participativo, o que pode ser feito por meio do levantamento tempestivo do índice de realização de demanda ao final da vigência de cada ata de registro de preços. Dessa forma, seria possível identificar tempestivamente as licitações com pior desempenho em relação à acurácia da previsão de demanda e buscar alternativas para melhorar o desempenho.

Por fim, sugere-se que a instituição reveja sua política de compras, passando a utilizar o Sistema de Registro de Preços apenas para as aquisições frequentes e parceladas. Dessa forma, itens que são adquiridos de uma única vez ou somente quando há recursos orçamentários disponíveis (materiais permanentes, por exemplo), passariam a ser licitados, na forma tradicional, a qual é inclusive mais rápida que o SRP e dá aos fornecedores a certeza de realização das demandas, o que permite que a Administração obtenha preços melhores (NUNES; DANTAS, 2012).

\section{CONSIDERAÇÕES FINAIS}

As inovações na administração pública são importantes no sentido de promover o melhor desempenho das instituições, o que contribui para o atendimento ao princípio 
constitucional da eficiência, refletindo em bem estar para o cidadão. Contudo, é necessário que a gestão das inovações implementadas seja feita no sentido de garantir que estas atinjam os objetivos para os quais foram criadas, o que demonstra a importância das atividades de monitoramento, controle e avaliação.

A presente pesquisa teve como objetivo geral analisar o planejamento participativo do IFTM, uma inovação feita pela instituição no intuito de tornar os itens e quantitativos licitados mais próximos da realidade, por meio do envolvimento de um maior número de pessoas. Contudo, os resultados da pesquisa apontam que o planejamento participativo não tem sido suficiente para promover uma maior acurácia da previsão de demanda, uma vez que não houve diferença significativa na comparação dos valores encontrados com os valores de referência.

Contudo, ressalta-se que os resultados encontrados para o índice de realização das demandas não estão diretamente relacionados ao envolvimento de um maior número de pessoas no processo de planejamento, mas sim à utilização inadequada do SRP, o qual, apesar de ser uma ferramenta de melhoria nos processos de planejamento e compras públicas, especialmente por permitir que a administração somente efetive as demandas quando e se precisar, necessita ter seu uso baseado em técnicas adequadas de previsão de demanda.

Diante dos resultados encontrados e com base na literatura pesquisada, alguns ajustes foram recomendados à instituição pesquisada, no sentido de promover melhorias no processo: adoção do índice do período anterior como subsídio para planejamento de quantitativos; conciliação dos valores licitados com o orçamento; capacitação dos servidores envolvidos; ações de monitoramento e controle da inovação implementada e revisão da política de uso do SRP na instituição.

Como limitações dessa pesquisa, destaca-se o fato de sua aplicação ter ocorrido em apenas uma instituição. Portanto, os resultados obtidos aqui, apesar de fornecerem importantes subsídios para gestores públicos, em especial aos da instituição pesquisada não podem ser generalizados. Vale destacar também que o estudo não abordou os índices de realização das demandas das licitações realizadas antes de 2013, ou seja, antes do planejamento participativo, não sendo possível verificar se houve melhora dos índices com a nova metodologia de planejamento. Além disso, como o foco da análise feita nessa pesquisa é a realização das demandas registradas, não foram observados outros aspectos do planejamento participativo, tais como impactos nas rotinas de trabalho e na satisfação dos profissionais 
envolvidos, aspectos nos quais o planejamento participativo pode ter apresentado resultado positivo.

No tocante a escala de análise utilizada, essa pode ser considerada uma boa ferramenta para o levantamento e acompanhamento dos índices de realização das demandas. Contudo, a nomenclatura das classes (muito insatisfatória, insatisfatória, regular, satisfatória e muito satisfatória) foi definida levando em conta somente a amplitude dos dados. Dessa forma, resultados próximos aos limites das classes deixam de ter seu valor absoluto considerado. Apesar disso, entende-se que não houve prejuízo à qualidade dos resultados da pesquisa, uma vez que a escala não foi a única forma de análise dos dados, os quais também foram submetidos ao teste de hipóteses.

Destaca-se que, embora os resultados aqui obtidos não possam ser generalizados para outras instituições, as sugestões feitas no presente trabalho no intuito melhorar o desempenho da inovação implementada podem ser adotadas por qualquer instituição pública que utilize o SRP, o que certamente contribuirá para uma maior acurácia da previsão de demanda e, indiretamente, na obtenção de melhores preços nas licitações. No tocante a inovação no setor público, o estudo evidenciou aos gestores a necessidade de se monitorar e avaliar adequadamente as inovações implementadas, a fim de se promover tempestivamente ajustes no intuito de se obter melhores resultados.

Sugere-se, em pesquisas futuras, estudos semelhantes em outras instituições, de modo a verificar se os resultados aqui encontrados se repetem nestas e no intuito de encontrar instituições com índices melhores, as quais podem ser usadas para subsidiar propostas de melhorias futuras. Sugere-se ainda a realização de pesquisas com foco no preço dos itens registrados no SRP, a fim de confirmar se a baixa realização das demandas tem impacto no aumento dos preços por parte dos fornecedores, hipótese não abordada na presente pesquisa.

\section{REFERENCIAS}

BRASIL. Decreto ${ }^{\circ} 7.892$, de 23 de janeiro de 2013. Regulamenta o Sistema de Registro de Preços previsto no art. 15 da Lei no 8.666, de 21 de junho de 1993. Diário Oficial da República Federativa do Brasil, Brasília, 24 jan. 2013.

BRASIL. Lei ${ }^{\circ}$ 8.666, de 21 de junho de 1993. Regulamenta o art. 37, inciso XXI, da Constituição Federal, institui normas para licitações e contratos da Administração Pública e dá outras providências. Diário Oficial da República Federativa do Brasil, Brasília, 22 jun. 1993. 
BRASIL. Tribunal de Contas da União. Relatório TC-017.599/2014-8. 2014. Disponível em: $<$ http://portal.tcu.gov.br/lumis/portal/file/fileDownload.jsp?fileId=8A8182A15072571D0150 813BF9AE5ACC> Acesso em: 5 set. 2018.

FENILI, Renato Ribeiro. Gestão de Materiais. Brasília: ENAP, 2015.

FERRAREZI, Elisabete; AMORIM, Sonia; TOMACHESKI, João. A sustentabilidade de iniciativas premiadas no Concurso Inovação: indícios de mudança da gestão no governo federal? Cadernos Enap, Brasília, n. 34, p. 11-51, 2010.

GIL, Antonio Carlos. Como elaborar projetos de pesquisa. 4. ed. São Paulo: Atlas, 2002.

GODOY, Arilda Schmidt. Pesquisa qualitativa: tipos fundamentais. Revista de

Administração de Empresas, São Paulo, v. 35, n. 3, p.20-29, jun. 1995.

HALVORSEN, Thomas; HAUKNES, Johan; MILES, Ian; RØSTE, Rannveig. On the differences between public and private sector innovation. Oslo: NIFU STEP, 2005. Disponível em

$<$ http://unpan1.un.org/intradoc/groups/public/documents/apcity/unpan046809.pdf $>$ Acesso em 5 set. 2018.

INSTITUTO FEDERAL DO TRIÂNGULO MINEIRO - IFTM. Resolução "ad referendum" n' 96/2013, de 26 de dezembro de 2013: Dispõe sobre a aprovação do Plano de Desenvolvimento Institucional 2014-2018, do Instituto Federal de Educação, Ciência e Tecnologia do Triângulo Mineiro. 2013. Disponível em:

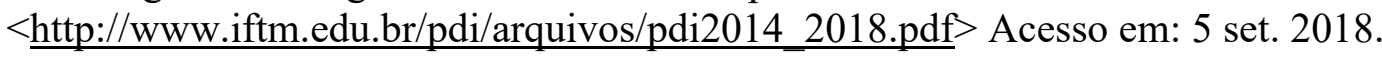

JACOBY FERNANDES, Jorge Ulisses. Sistema de Registro de Preço e pregão presencial e eletrônico. 5. ed. rev. atual. e ampl. Belo Horizonte: Fórum, 2013.

KOCH, Per; HAUKNES, Johan. On innovation in the public sector - today and beyond.

Publin Report. Oslo, NIFU-Step, 2005. Disponível em:

$<$ https://brage.bibsys.no/xmlui/bitstream/handle/11250/226573/d20-

innovation.pdf? sequence $=1$ \&isAllowed $=\mathrm{y}>$ Acesso em: 5 set. 2018.

LUCHI, Juliana Silva Prado; CARNEIRO, Teresa Cristina Janes. Análise da eficiência e eficácia do Pregão Presencial. In: Encontro Nacional de Engenharia de Produção, 26, 2006, Fortaleza. Anais... Salvador: ABEPRO, 2006.

NUNES, André; DANTAS, Lucimar de Oliveira. Eficiência do sistema de registro de preços: um estudo de caso do Superior Tribunal de Justiça. Universitas: Gestão e TI, Brasília, v. 2, n. 1, p.1-10, 3 ago. 2012.

OLIVEIRA, Luiz Guilherme de. Inovação no setor público: uma reflexão a partir das experiências premiadas no Concurso Inovação na Gestão Pública Federal. Brasília: ENAP, 2014. 
ORGANIZAÇÃO PARA COOPERAÇÃO E DESENVOLVIMENTO ECONÔMICO OCDE. Manual de Oslo: Diretrizes para coleta e interpretação de dados sobre inovação. 3. ed. Paris: OCDE, 2005.

PAIM TERRA, Antonio Carlos. Compras públicas inteligentes: uma proposta para a melhoria da gestão das compras governamentais. 2018. Disponível em:

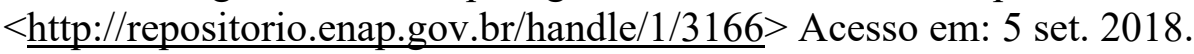

PARANÁ. Lei n 17.081, de 09 de fevereiro de 2012. Dispõe sobre normas para as compras públicas pelo sistema "Registro de Preço", na forma que especifica. Diário Oficial Executivo, Curitiba, 17 fev. 2012.

PEIXOTO, Graziani Mendonça. Previsão de demanda em registro de preços: análise dos processos de compras da Reitoria do Instituto Federal do Espírito Santo. 2016. $174 \mathrm{f}$. Dissertação (Mestrado) - Curso de Gestão Pública, Centro de Ciências Jurídicas e Econômicas, Universidade Federal do Espírito Santo, Vitória, 2016.

PEREIRA JÚNIOR, Jessé Torres; DOTTI, Marinês Restelatto. O manejo do registro de preço e o compromisso com a efíciência. Revista do TCU, Brasília, n. 118, p. 65-76, mai./ago. 2010 .

PINHEIRO, Keite Mendonça; BOSELLI, Felipe. Registro de Preços: a aplicação deste sistema na visão do fornecedor à Administração Pública. Revista Empreendedorismo e Sustentabilidade, São José, v. 1, n. 1, p.140-158, 2016.

RAPOSO, Matheus Hortas et al. A importância do planejamento de compras para a gestão estratégica de suprimentos. In: DEZOLT, Ana Lúcia et al. (Org.). Compras públicas estaduais: Boas práticas Brasileiras. Brasília: CONSAD, 2016. p. 204-225. Disponível em: $<$ http://consad.org.br/wp-content/uploads/2016/06/Livro-Boas-praticas-de-compras-publicasv-final-15.07.2016.pdf >. Acesso em: 5 set. 2018.

SANTOS, Nathália de Melo. Inovação no setor público: estado da arte e proposição de uma agenda de pesquisa. In: Encontro da ANPAD, 41, São Paulo. Anais... ANPAD, 2017.

SCHERER, Felipe Ost. Gestão da inovação no setor público: a metodologia do octógono da inovação no setor público. 2014. Disponível em:

$<$ https://www.tjrs.jus.br/export/poder_judiciario/tribunal de justica/centro de estudos/horizo ntes/inovacao do setor publico.pdf>. Acesso em 5 set. 2018.

SIEGEL, Sidney; CASTELLAN JR., N. John. Estatística não paramétrica para ciências do comportamento. 2. ed. Porto Alegre: Artmed, 2006.

TEIXEIRA, Augusto César. Os processos de planejamento estratégico e controle orçamentário de uma instituição de educação superior, básica e profissional: o caso IFTM. 2017. Dissertação (Mestrado) - Curso de Pós-graduação em Gestão Organizacional, Faculdade de Gestão e Negócios, Universidade Federal de Uberlândia, Uberlândia, 2017.

VEIGA, Halisson Golffetto da. Previsão de demanda em Registros de Preços: Análise do Departamento de Gestão de Restaurantes da Universidade Federal do Espírito Santo. 2015. 
Dissertação (Mestrado) - Curso de Pós-graduação em Gestão Pública, Centro de Ciências Jurídicas e Econômicas, Universidade Federal do Espírito Santo, Vitoria, 2015.

VERGARA, Sylvia Constant. Projetos e Relatórios de Pesquisa em administração. 8. ed. São Paulo: Atlas, 2007.

VERGARA, Sylvia Constant. Métodos de Pesquisa em Administração. 6. ed. São Paulo: Atlas, 2016.

VRIES, Hanna de; BEKKERS, Victor; TUMMERS, Lars. Innovation in the public sector: a systematic review and future research agenda. Public Administration, [s.i.], v. 94, n. 1, p.146-166, 2015 